\title{
Stability and startup of non linear loop circuits
}

\author{
Francesca Cucchi, Stefano Di Pascoli`, and Giuseppe Iannaccone \\ Dipartimento di Ingegneria dell'Informazione \\ University of Pisa \\ Via Caruso 16, 56122 Pisa, Italy
}

\begin{abstract}
The reliable analysis of DC operating point in circuits with positive feedback topology is often challenging, and frequently performed with ad hoc methods. These techniques are often error prone and lead to the frequent use of sub-optimal or unnecessary additional circuits for the stabilization or determination of the operating point (startup circuits). We present a simple and reliable technique for the determination of "stable" circuit solutions, that is based on the use of available circuit simulators and hence takes advantage of accurate device models. The method has been experimentally validated on a self-biasing current generator fabricated with a standard $0.18 \mu \mathrm{m}$ CMOS process.
\end{abstract}

Keywords: Self Biasing, Operation Point, Analog Circuits

\section{Introduction}

In the realm of electronic circuits containing active devices, the determination of the operating point is a basic step of the design process. It is one of the few engineering techniques requiring the solution of an inherently non-linear physical system. Since non-linear systems cannot generally be solved in closed form, the electronic designer has to resort to approximate solutions, numerical analysis tools or, sometimes, clever ad hoc tricks. In fact, this intrinsic non-linearity is seldom a problem, since most circuits are designed to have an operating point that can be easily determined.

However, some applications demand the use of circuits for which the computation of the operating point is non trivial. The typical case is a circuit with a positive feedback such as the well known Eccles-Jordan flip-flop. These circuits can have a few operating points, some of which "unstable". Due to the mentioned non-linearity, the analysis of these circuits can be challenging; furthermore, in this case commonly used circuit simulators, such as SPICE, often provide unreliable information, since they can converge to the "unstable" solution.

General methods have been developed for the non-linear analysis of active circuits[1-3], but are generally too abstract, provide poor physical insight on circuit operation, and are of little help to the circuit designer. As a consequence, non-linear circuits are usually analysed with simple pencil and paper methods[4].

\footnotetext{
* Corresponding author: stefano.di.pascoli@unipi.it
} 

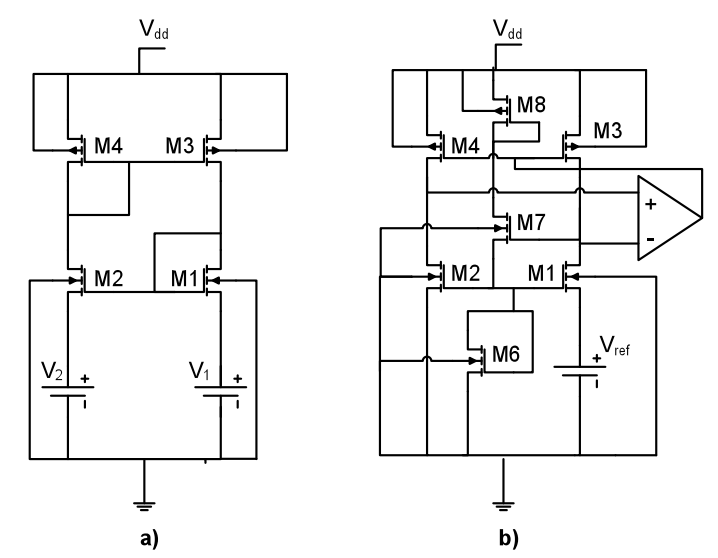

Fig. 1. Self-biased current generator: simplified proof of concept circuit (a) and complete circuit (b); M6, M7 and M8 are needed to set the bias point of M1 (a native transistor with negative threshold voltage); the operational amplifier imposes $V_{D S_{M 3}}=V_{D S_{M 4}}$ improving the accuracy of the upper current mirror; since in the complete circuit $V_{2}=0$ no generator is connected in series with M2.

These calculations are constrained to the use of crude first-level device models, which can lead to grossly approximated solutions, missed solutions and also to spurious solutions. Another common way to investigate the stability properties of circuits is the use of (time consuming) transient simulations, but these can also provide unreliable information in case of circuits with widely separated time constants (ill-conditioned systems). In order to overcome these shortcomings, we propose a method that is able to find the operating points and the stability properties of many commonly used non-linear feedback circuits.

\section{Problem definition}

A non-linear time-independent circuit (i.e., without capacitors and inductors) can be described with a system of equations $F(\mathbf{x})=\mathbf{0}$, where the vector $\mathbf{x}$ is composed by node voltages and/or branch currents. The system can have an unknown number of solutions $\mathbf{x}_{\mathbf{i}}$. Most circuits have only one solution, but circuits with more than one solution are well known. Eccles-Jordan circuits generally have three solutions, one of which is "unstable".

We must note that even the "stability" of the solution is not a well-defined concept. Solutions of time-independent circuits cannot be "stable" or "unstable". Indeed, unstable solution are not solutions at all. A formal definition of "stable solution" can be found in [5]: a solution of $F(\mathbf{x})=\mathbf{0}$ is potentially stable if it is possible to build - adding capacitors between nodes and inductors in series to the branches of the given circuit - an augmented circuit which is robustly stable in the time domain. Robustly stable means that the stability is not compromised by the addition of another set of sufficiently small capacitors and inductors to 
the given circuits (i.e. the values of the first set of capacitors and inductors must not be critical). Solutions which are not potentially stable are unstable.

Many non-linear circuits with more than one solution are based on a positivefeedback loop topology, like, for example, self-biased current generators, in which two current-controlled current generators are connected back-to-back in a positivefeedback loop. We will take this circuit as an example for illustrating the method (Fig.1a).

Transistors M3 and M4 form a linear current mirror, duplicating the current fed into the drain of M4 ( $\left.I_{\text {in_um }}\right)$ onto the drain of M3 (Iout_um $)$. This current mirror provides a linear relationship between its input and output:

$$
I_{\text {out_um }}=k_{u m} I_{\text {in_um }},
$$

where $k_{u m}$ depends on the geometry of M3 and M4. On the other hand, the lower mirror (M1, M2, V1, and V2) provides a nonlinear relationship between the input current (the drain current of M1, $I_{\text {in_lm }}$ ) and the output (the drain current of M2 $\left.I_{\text {out_lm }}\right)$ :

$$
I_{\text {out_lm }}=f\left(I_{\text {in_lm }}\right) .
$$

The ratio of the input to the output current $k_{l m}$ depends on the input current. At equilibrium we must have

$$
k_{u m}=1 / k_{l m} .
$$

If $k_{l m}$ is a monotonic function of the input the (3) can be satisfied for a single set of circuit currents. However, as [4] points out, both mirrors of the circuit provide zero current when fed with a zero input and hence another equilibrium point exists, with all null currents (where $k_{l m}$ is undefined). For this reason most designers of self-biased current generators include a startup circuit which forces the circuit to the desired solution, avoiding the zero-current one [6-8].

However, the above discussion is oversimplified. Simulating the circuit (with a UMC .18 $\mu \mathrm{m}$ CMOS technology, and with identically sized M3 and M4) we find that if $\beta_{1}>\beta_{2}$ and $V_{1}>V_{2}$, where $\beta_{i}=\mu C_{o x} W_{i} / L_{i}\left(W_{i}\right.$ and $L_{i}$ are transistor width and length, $\mu$ is carrier mobility and $C_{o x}$ is the gate oxide capacitance per unit area) are referred to transistors $M_{i}$, the circuit undergoes a transient ending at the equilibrium point with non-zero currents. Hence, no startup circuit seems required. Instead, if $\beta_{1}<\beta_{2}$ and $V_{1}<V_{2}$ the circuit never settles in the equilibrium point suggested by eq.(3), and no startup circuit can help. For the other possible configurations $\left(\beta_{1}<\beta_{2}\right.$ and $V_{1}>V_{2} ; \beta_{1}>\beta_{2}$ and $\left.V_{1}<V_{2}\right)$ eq. (3) is never verified and no equilibrium point is possible.

\section{Proposed solution}

To solve this problem we developed a technique that provides valuable information on the equilibrium points of nonlinear circuit. If we can consider a nonlinear circuit as a closed loop of nonlinear blocks (Fig. 2a), we can cut open the loop 
and insert the circuitry shown in Fig. 2b. Even if the method can be adapted to cuts in any branch, we will discuss only the most useful case, when the current flowing in the severed branch is non zero. The case of zero current is indeed simpler, but less general. The independent current source sends in the circuit a test current $I_{t}$ which gives rise to a voltage $V_{p}$ across its terminals. The voltagecontrolled generator imposes the same voltage $V_{p}$ to node $B$, the other end of the cut loop. Obviously, when the current $I_{v}$ sinked by the voltage generator is equal to $I_{t}$, the original uncut circuit is in equilibrium. The two sides of the cut could be directly connected without altering the branch currents and the node voltages. Hence if we plot $I_{v}$ vs. $I_{t}$, equilibrium points can be identified as the intersections between the $I_{v}\left(I_{t}\right)$ curve and the $I_{v}=I_{t}$ line. In addition, the derivative $\partial I_{v} / \partial I_{t}=\lambda$ at the equilibrium point enables us to determine the stability of the equilibrium point.

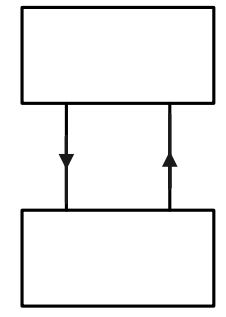

a)

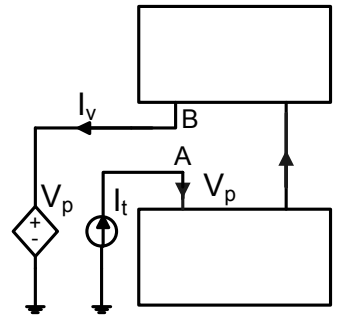

b)

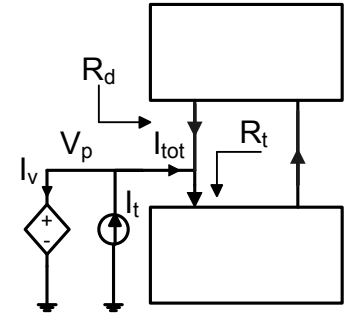

c)

Fig. 2. Non-linear loop analysis

Let us call $R_{t}$ the differential resistance seen by the $I_{t}$ generator: if the test current increases by $\Delta I_{t}$, the voltage $V_{p}$ increases by $\Delta V_{p}=R_{t} \Delta I_{t}$. The current $I_{v}$, instead, increases by $\Delta I_{v}=\lambda \Delta I_{t}$. Since the nodes $A$ and $B$ are at the same voltage, we connect them and redraw the circuit as in Fig. 2c. The total differential resistance seen between nodes $A \equiv B$ and ground (as shown in Fig. 2c) can be written as:

$$
R_{d}=\frac{\Delta V_{p}}{\Delta I_{t o t}}=\frac{R_{t} \Delta I_{t}}{\Delta I_{t}-\lambda \Delta I_{t}}=\frac{R_{t}}{1-\lambda}
$$

where $\Delta I_{\text {tot }}$ is indicated in Fig. 2c. From (4) we can conclude that if $\lambda>1$ this solution is unstable. Let us underline that we assumed $R_{t}>0$, which is the typical situation is practical circuits, but the method can in theory be easily generalized to any initial sign of $R_{t}$. Furthermore, $\lambda$ is the small-signal DC loop gain, and hence the fact that values in excess of 1 lead to instability is well known.

Hence, the practical application of the method consists of cutting open a loop, inserting the proper generators and performing a DC simulation of the circuit with an input current sweep. The analysis of circuit Fig. (1a) (for which 

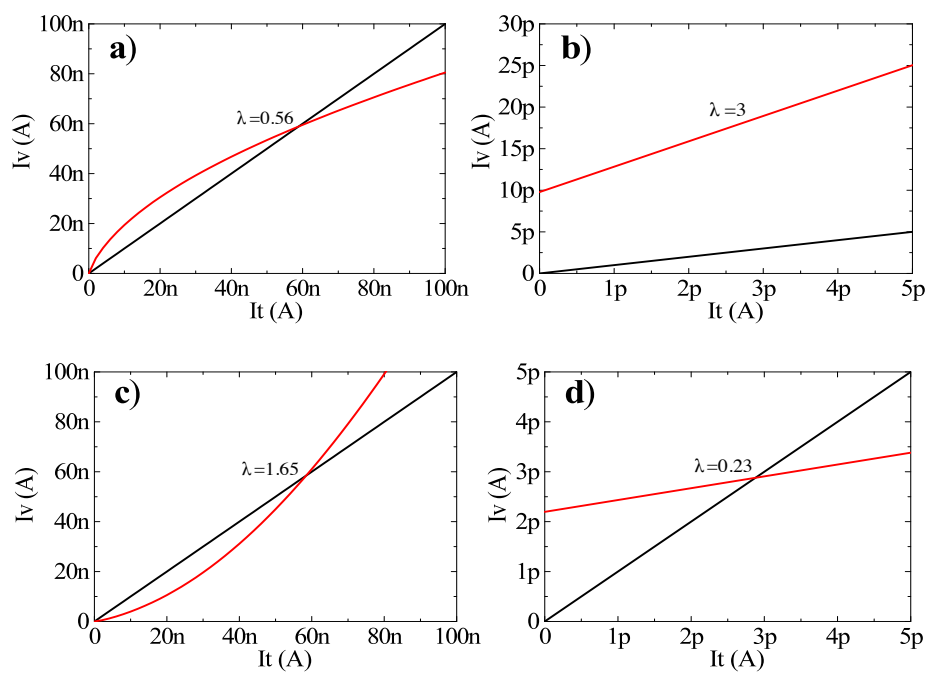

Fig. 3. SPECTRE dc sweep of circuit of Fig. 1, cut at the drain of M3: current generator to gate-drain of M1, voltage generator to M3 drain. $\beta_{1}>\beta_{2}$ and $V_{1}>V_{2}$ (a); particular of low current region (b); $\beta_{1}<\beta_{2}$ and $V_{1}<V_{2}$ (c); particular of low current region (d) ( $\lambda$ is the derivative of the current at the intersection; the black straight lines are $I_{V}=I_{t}$, while the red lines show the simulation results.

is $R_{t}>0$ ) leads to the results of Figures 3a-b, which show that a single and stable operating point is obtained only for $\beta_{1}>\beta_{2}$ and $V_{1}>V_{2}$. It is worth noticing that in this case no equilibrium point exist at $I_{t}=0$ and hence no startup circuitry is needed. Figures 3c-d show instead that for $\beta_{1}<\beta_{2}$ and $V_{1}<V_{2}$ the solution is unstable, and another stable solution is present for very small currents. Therefore, with the use a circuit simulator equipped with accurate device models we can learn that often some pencil-and-paper results, such as the zero-current stable solution, can indeed be artifacts due to the use of too simplistic device models.

Furthermore, this approach provides valuable physical insights on the circuit. Since the $I_{v}\left(I_{t}\right)$ relationship provided by the simulations can be interpreted as the input-output characteristic of an amplifier, a designer can usually devise modifications to the circuit which can modify it in a foreseeable manner. Hence, the above analysis not only can provide evidence of bias or stability problems, but is also a tool for their solution.

The circuit of Fig. 1(b) has been designed and fabricated, using native transistors (with threshold voltage $<0$ ) for M1 and M2. In this version of the circuit M1 was not diode-connected and a proper bias circuit was added in order to bias M1 in saturation. $V_{1}$ and $V_{2}$ were set to $335 \mathrm{mV}$ and 0 , respectively. Using the proposed method, we obtained the results of Fig. 4(left). The current in M1 is about $7 \mathrm{nA}$, and the operating point is stable. This is confirmed by measurements 

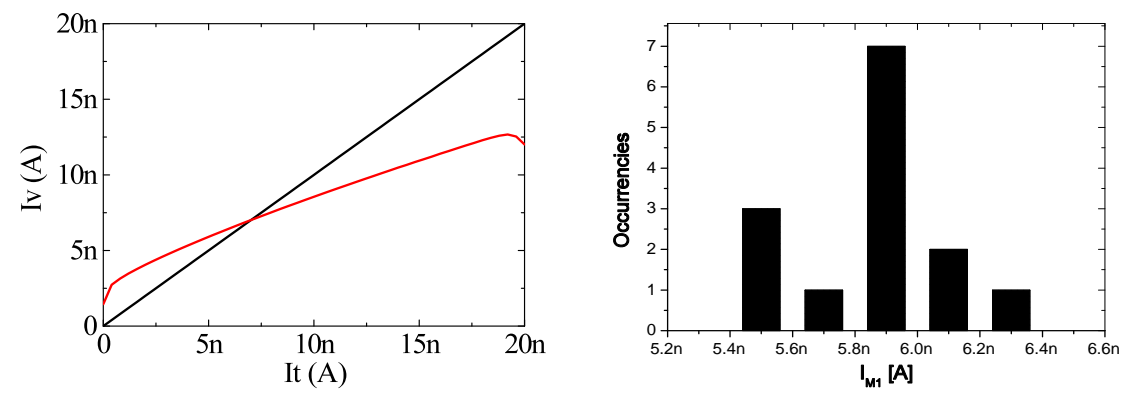

Fig. 4. $I_{v}$ vs. $I_{t}$ for the complete circuit of Fig. 1(b) (left) and $I_{M 1}$ distribution in 14 samples of the Fig.1(b) circuit (right).

on 15 samples realized in a $0.18 \mu \mathrm{m}$ UMC CMOS technology. Fig.4(right) shows the current distribution in 14 working samples; the mean current is $5.85 \mathrm{nA}$ $(\sigma=0.24 \mathrm{nA})$ and no start up problems were observed.

\section{Acknowledgement}

This work has been partially supported by the Electronic Components and Systems for European Leadership Joint Undertaking and by the Italian Ministry of Education, University and Research (MIUR) under grant agreement No 737434 (CONNECT).

\section{References}

1. Chua,L.,Green D.: A Qualitative Analysis of the Behavior of Dynamic Nonlinear Networks: Stability of Autonomous Networks. IEEE Trans. Circuits Syst. 23, 355379 (1976)

2. Green M., Wilson A.: An Algorithm for Identifying Unstable Operating Points Using SPICE. IEEE Trans. Comput.-Aided Design Integr. Circuits Syst. 14, 360-370 (1995)

3. Storti Gajani G., Brambilla A., Premoli A.: Numerical Determination of Possible Multiple DC Solutions of Nonlinear Circuits. IEEE Trans. Circuits Syst. 55, 10741083 (2008)

4. Sedra A., Smith K.: Microelectronic Circuits. Oxford University Press, New York (1997)

5. Green M., Willson A.: How to Identify Unstable dc Operating Point, IEEE Trans. Circuits Syst. 39, 820-832 (1992)

6. Guo J., Leung K.N. A CMOS voltage regulator for passive RFID tag ICs, Int. J. Circ. Theor. App. 40, 329-340 (2012)

7. Liang C.J., Chung C.C.: Lin H. A low-voltage band-gap reference circuit with second-order analyses. Int. J. Circ. Theor. App. 39,1247-1256 (2011)

8. Tsitouras A., Plessas F., Birbas M., Kikidis J., Kalivas G.: A sub-1V supply CMOS voltage reference generator. Int. J. Circ. Theor. App. 40, 745-758 (2012) 